\title{
Erratum to: Microsatellite loci for a new Australian endemic plant species Erythroxylum sp. 'Cholmondely Creek' (J.R.Clarkson 9367) (Erythroxylaceae)
}

Marlien van der Merwe · Joelle Catherine • Maurizio Rossetto

Published online: 26 January 2011

(C) Springer Science+Business Media B.V. 2011

Erratum to: Conservation Genet Resour (2009) 1:329-331

DOI 10.1007/s12686-009-9076-x

The second authors name should read Joelle Catherine and not Joelle Christina.

The online version of the original article can be found under doi:10.1007/s12686-009-9076-x.

M. van der Merwe $(\bowtie) \cdot$ J. Catherine $\cdot$ M. Rossetto National Herbarium of New South Wales, Botanic Gardens Trust, Mrs Macquaries Rd, Sydney, NSW 2000, Australia e-mail: marlien.vandermerwe@ @rbgsyd.nsw.gov.au; marlien.vandermerwe@gmail.com 